\title{
Modelling fibrillation potentials - Analysis of time parameters in the muscle intracellular action potential
}

\author{
Javier Rodríguez Falces ${ }^{1}$, Armando Malanda Trigueros ${ }^{1}$, Luis Gila Useros ${ }^{2}$, Ignacio Rodríguez Carreño ${ }^{1}$, Javier Navallas Irujo ${ }^{1}$
}

(1) Universidad Pública de Navarra D.I.E.E. Campus de Arrosadía s/n. 31006 Pamplona. Spain. Tel:+34 948169312.

Fax: +34948169720 e-mail: malanda@unavarra.es

(2) Hospital Virgen del Camino Dpto. Neurofisiología Clínica. Tel: +34 948 429475. e-mail: lgilau@ yahoo.com

\begin{abstract}
A single fibre action potential (SFAP) can be modelled as the convolution of a biolectrical source and a filter impulse response. In the Dimitrov-Dimitrova (D-D) convolutional model, the first temporal derivative of the intracellular action potential (IAP) is used as the source, and $T_{\text {spl }}$ is a time parameter related to the duration of the IAP waveform. Our work is centred on the relation between Tspl and the main spike duration (MSD), defined as the time interval between the first and third phases of the SFAP. We show that Tspl essentially determines the MSD parameter. As experimental data, we used fibrillation potentials (FPs) of two different muscles to study the D-D model. We found that $T_{\text {spl }}$ should have a certain statistical variability in order to explain the variability in the MSD of our FPs. In addition, we present a method to estimate the $T_{\text {spl }}$ values corresponding to a given SFAP from its measured MSD.
\end{abstract}

\section{INTRODUCTION}

It is generally assumed that a single muscle fibre action potential (SFAP) can be modelled as a convolution of a source and a transfer function [1]-[2]-[3]. In the DimitrovDimitrova (D-D) SFAP model, the first temporal derivative of the intracellular action potential (IAP) is used as the source.

In terms of volume conductor theory [4]-[5]-[6]-[3], SFAPs are triphasic waveforms. The dependences of SFAP amplitudes $V_{1}, V_{2}, V_{3}$ and $V_{\mathrm{pp}}$ (Fig. 1) on radial distance, $r$ have been characterized by several authors [7]-[8]-[9]. As the radial distance increase, generally the amplitude decreases while the time parameters of the SFAP main spike (i.e. UT, DT, MSD, DF25, DS25 and SP10 in Fig. 1) increase. Of the various time parameters of a SFAP, in this study we focus our attention on the main spike duration (MSD) defined as time interval between the peaks $V_{1}$ and $V_{3}$ (Fig. 1). The MSD can be divided into two portions: the first one, which we call the up-time (UT), extends between $V_{1}$ and $V_{2}$ and the second one, which we call the decay time (DT), extends between $V_{2}$ and $V_{3}$.

This paper follows a previous study about the D-D model, concerning the peak-to-peak ratio of a SFAP (ratio between $\mathrm{V}_{2}$ and $\mathrm{V}_{1}$ ) [10]. Here we compare the MSD figures of the D-D model [2] with those of a set of recorded fibrillation potentials (FPs). The MSDs obtained from FPs vary considerably from one muscle to another and also from fibre to fibre within the same muscle.

In the D-D model, $T_{\text {spl }}$ is a time parameter that determines the profile of the repolarization of the intracellular action potential (IAP), which in turn determines the duration of the IAP waveform. We will show that the MSD of a SFAP is strongly dependent on the $T_{\text {spl }}$ parameter of its corresponding IAP. In fact, if $T_{\mathrm{spl}}$ is kept constant the
SFAPs that can be generated following the D-D model present only slight variations in their MSDs. We should therefore permit $T_{\mathrm{spl}}$ to vary within certain limits in order to generate the wide MSD range observed in experimental recordings.

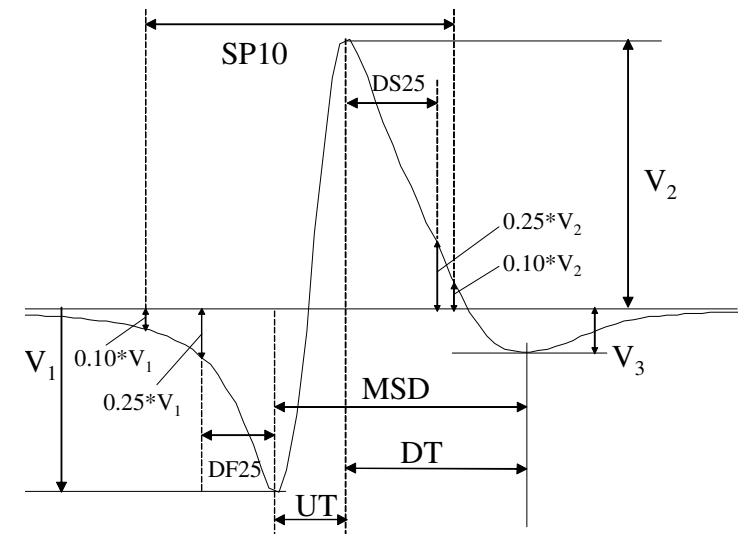

Fig. 1 Typical SFAP waveform: $V_{1}, V_{2}$ and $V_{3}$ are the amplitudes of the first, second and third phases, respectively. Six time parameters are defined in the figure: UT is the up-time (which extends between peaks $V_{1}$ and $V_{2}$ ), DT is the decay time (which extends between peaks $V_{2}$ and $V_{3}$ ), MSD is the main spike duration (which extends between peaks $V_{1}$ and $V_{3}$ ), DF25 (time interval between $0.25 \cdot V_{1}$ and the peak $V_{1}$ ), DS25 (time interval between $V_{2}$ and $0.25 \cdot V_{2}$ ) and SP10 (time interval between $0.10 \cdot V_{1}$ and $\left.0.10 \cdot V_{2}\right)$.

The study of $T_{\mathrm{spl}}$ is focused on the improving of the IAP mathematical model. Other authors have already reviewed the repolarization phase of the IAP [11]-[12]-[13]-[14][15]. Several features of the FPs have been studied in some pathological conditions [15], but, as it has pointed out by other authors [14], further work is needed to prove the diagnostic utility of the SFAP's subcomponents analysis in neuromuscular disorders.

The propagation velocity and fibre diameter are two parameters mathematically interrelated in the D-D impulse response [1]. The influence of these parameters on the SFAP MSD is studied in detail. We will show that, regarding the D-D model, the variation in the diameter (and therefore, in the propagation velocity) of the denervated muscle fibres has little relevance on the MSD.

Our study has two different goals which are addressed in two sequential stages. Firstly, we establish the variability that has to be introduced in the $T_{\mathrm{spl}}$ parameter to accommodate, in a statistical way, the characteristics of real FPs in synthetic SFAPs. Secondly, we derive a method to estimate the $T_{\text {spl }}$ value corresponding to a given SFAP from the MSD measured in the SFAP. 
In Section 2 of this paper, we analyze our bank of FPs and present histograms of their corresponding MSDs. In Section 3, we examine the influence of the D-D model parameters on the MSD and discuss the necessity of introducing variability in the $T_{\mathrm{spl}}$. In Section 4 , we present a method for estimating the $T_{\text {spl }}$ value of an IAP from the MSD value of the corresponding observed SFAP. $T_{\text {spl }}$ histograms from the experimental recordings are calculated and final conclusions are given.

\section{Fibrillation potentials as experimental SFAPs}

\subsection{Test signals}

The signals were recorded with an electromyograph (Counterpoint, Dantec Co., Skovlunde, Denmark) using conventional concentric needle electrodes (type DCN37; diameter $=0.46 \mathrm{~mm}$, recording area $=0.07 \mathrm{~mm}^{2}$; Dantec Co.). A band-pass filter (low and high frequencies of $20 \mathrm{~Hz}$ and $10 \mathrm{kHz}$, respectively) was used before the signal was sampled at a rate of $25.6 \mathrm{kHz}$ and digitalized at 16 bits persample. Data were stored on the hard disk of a PC computer. Further analysis of recorded signals was performed off-line on a PC computer using a software tool developed by us and implemented with the Matlab 4.2c.1 and the Signal Processing Toolbox 3.0b.

FPs were extracted from two acute, partially-denervated muscles. One was a right abductor digiti minimi (ADM) muscle of a 28-year-old man whose ulnar nerve had been sectioned at the elbow in an accident involving a glass sharp end a month previous to the electromyographic study. The other was a right extensor digitorum communis (EDC) muscle of a 72-year-old woman with a radial nerve lesion due to fracture of the humerus three months before the electromyographic exploration.

FPs were extracted from 3 different insertion points in the $\mathrm{ADM}$ and from 4 insertion points in the EDC. The position of the concentric needle electrode was changed several times for each insertion point in order to locate FPs. From all the FPs registered, those with bi or triphasic morphology and with at least three undistorted discharges were selected for further analysis. Up to 20 consecutive triggered discharges of each FP were extracted. A total of 370 different potentials, identified as FPs by an expert neurophysiologist, were accepted for analysis from the ADM muscle and 350 from the EDC. The signals are indeed fibrillation potentials (and not motor unit action potentials) since the patients did not make any voluntary contraction to generate them.

A small comment is worth about the selection of our filters. According to the definition of the parameter MSD, FPs are supposed to be triphasic. Although we used a high pass cut-off filter frequency of $20 \mathrm{~Hz}$, the terminal phases of our FPs were not significantly distorted. If our goal had been to thoroughly study the morphology of the terminal phase of the FPs we should have used a high pass filter with a lower cut-off frequency (between $1-3 \mathrm{~Hz}$ ) in order to record this phase of the fibrillations more accurately. However, our goal was to measure the MSD distribution of our fibrillations, for which a $20 \mathrm{~Hz}$ cut-off frequency is sufficient to record FPs with a distinctively third phase.

\subsection{MSD histograms}

Fig. 2 presents the MSD histograms for the two muscles under study.
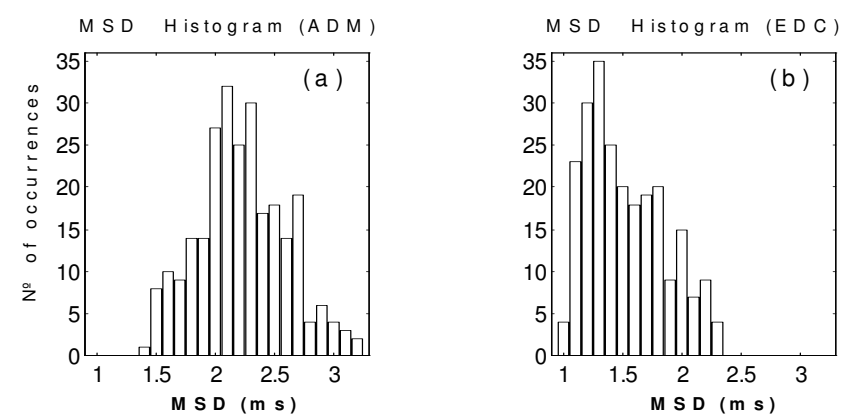

Fig. 2 MSD histograms for the ADM (a) and EDC (b). MSD histograms are centred around a different value for the ADM and EDC (2.1 and 1.3 ms, respectively). The dispersion around the most frequent $T_{\text {spl }}$ value is also different for each muscle (0.37 and 0.32 for the ADM and EDC, respectively).

The MSD histograms (Fig. 2) show the following features:

- The most frequent MSD values (mode) for the $\mathrm{ADM}$ and EDC muscles are $2.1 \mathrm{~ms}$ and $1.3 \mathrm{~ms}$, respectively.

- Values range from 1.4 to $3.2 \mathrm{~ms}$ in the ADM muscle and from 1.0 to 2.3 in the EDC. Therefore, the whole range for the ADM $(1.8 \mathrm{~ms})$ is clearly larger than that for the EDC (1.3 ms).

- The standard deviation (std) is large for both muscles, and larger for the ADM $(0.37 \mathrm{~ms})$ than for the EDC (0.32 ms).

\subsection{Radial distance}

It is useful to know the radial distances at which the FPs were recorded. With this information, the analysis of the D$\mathrm{D}$ model can be restricted to the radial distances of interest and the complexity of the $T_{\text {spl }}$ study can be reduced.

It is not possible to obtain directly the radial distance of a certain FP. Instead, a model-based indirect method was applied, similar to the analysis-synthesis approach used in voice processing for the analysis of speech segments [16]. More specifically, we searched for a SFAP with amplitude and spike duration as close as possible to that of the FP. The radial distance of the encountered SFAP was taken as an estimate of the radial distance of the FP. By following this procedure, we determined that a high percentage of the FPs were recorded within the $0.1-0.3 \mathrm{~mm}$.

If we assume a certain degree of variability in the diameter and the conduction velocity of the fibres of a muscle, then an enlargement of the latter range should be provided. To take this into account a range of 0.05-0.4 mm for the radial distance is used through the course of our study.

\subsection{MSD - radial distance correlation}

Neglecting some side effects (the frequency-dependence of some volume conduction parameters [17]-[18] or the distortion of the electric field introduced by the recording electrode [8]), we can assume that the amplitude of a SFAP is lower as the radial distance increases. In contrast, 
duration parameters of the SFAP main spike (i.e. UT, DT, MSD, DF25, DS25 and SP10 in Fig. 1) increase with radial distance. Thus, amplitude and duration parameters should present a degree of correlation as they both depend on the radial decline.

However, the correlation between the MSD parameter and the total amplitude $V_{\mathrm{pp}}$ is rather small for both muscles, specifically 0.18 for the ADM and -0.24 for the EDC. The variability in the fibre diameter and conduction velocity could explain low figures of correlation but they cannot justify a completely lack of correlation between MSD and $V_{\mathrm{pp}}$. In order to explain the low dependence of MSD on the radial distance it seems reasonable to look for some other parameter which affects the MSD in the D-D model.

\section{Analysis of the D-D model}

If we consider the fibre as a time-shift invariant system, the SFAP can be expressed as a convolution of two time dependent functions (1). In the D-D model, the input signal is the IAP 1 st temporal derivative, $\partial(\operatorname{IAP}(t)) / \partial t$, and the system impulse response, $\partial(1 / R(v t, r)) / \partial t$, is the potential generated at a recording point by two propagating unitary sources.

$$
\Phi_{e}(t)=C_{a n} \cdot \frac{\partial \operatorname{IAP}(t)}{\partial t} * \frac{\partial\left(\frac{1}{R(v t, r)}\right)}{\partial t}
$$

where $C_{\mathrm{an}}$ is a coefficient of proportionality, $v$ is the propagation velocity and $r$ the radial distance. The analytic expression of the impulse response is

$$
D D(r, v t)=\frac{v \cdot\left(z_{0}-v t\right)}{\left[\left(z_{0}-v t\right)^{2}+k_{a n} \cdot r^{2}\right]^{\frac{3}{2}}}
$$

where $z_{0}$ is the axial distance and $K_{\text {an }}$ the constant of anisotropy. (Here only the source closer to the electrode is considered). A mathematical dependence between $v$ and $d$ is well established [1]:

$$
v=v_{\text {med }}+0.05 \cdot(d \cdot 1000-55)
$$

In the next section we will study the dependence of the SFAP MSD on several D-D model parameters. These parameters are related either to the excitation or to the impulse response. We will study them separately for sake of clarity.

\subsection{Excitation parameters for the D-D model. Default values and ranges of variation}

Dimitrov and Dimitrova proposed an IAP approximation that provides for independent changes of different phases [2]. The time course of a typical IAP with a negative afterpotential is divided into four portions: the rising phase, the rapidly falling phase, the transition phase and the slowly falling phase (Fig. 3).
The first phase (the rising phase) is characterized by three parameters, namely $A_{1}, A_{2}$ and $A_{3}(4)$.

$$
R P(\tau)=A_{1} \cdot \tau^{A_{2}} \cdot e^{-A_{3} \tau} \quad 0 \leq \tau \leq \tau_{m}
$$

This phase models the depolarization occurring at the membrane. In the proximity of the fibre (from 0.05 to 0.4 $\mathrm{mm}$ away), the portion of the IAP profile that produces the most important part of the extracellular potential (including its positive and negative maxima) is the rising phase, i.e. the steeper phase of the profile [19]. Its duration $D_{1}$ (Fig. 3) is given by:

$$
D_{1}=\tau_{m}=\frac{A_{2}}{A_{3}}
$$

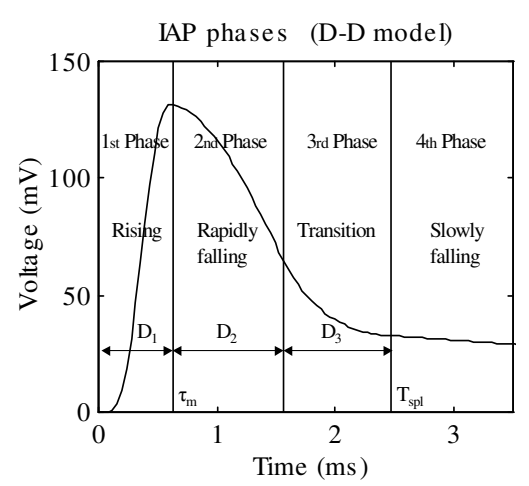

Fig. 3 Phases of the D-D intracellular action potential. The durations of the first three phases are $D_{1}, D_{2}$ and $D_{3}$, respectively. $T_{\text {spl }}$ is the sum of the duration of the three phases and determine the most relevant portion of the IAP.

The second, third and fourth IAP portions model the repolarization process occurring at the membrane of the cell after the depolarization. The durations of the second and third portions are respectively $D_{2}$ and $D_{3}$ (also shown in Fig. 3)

$$
D_{2}=D_{3}=\frac{T_{s p l}-D_{1}}{2}
$$

The duration of the rising phase (depolarization portion) of the IAP is fixed by parameters $A_{2}$ and $A_{3}(5)$, whereas the duration of the second and third phases are determined by the $T_{\text {spl }}$ parameter and $D_{1}$ (i.e. parameters $A_{2}$ and $A_{3}$ ) (6). Since $D_{1}$ is generally much smaller than $T_{\text {spl }}$, (and assuming that $D_{1}$ does not have a pronounced variation) the duration of the second and third phases are essentially determined by $T_{\text {spl. }}$.

Thus, the $T_{\text {spl }}$ parameter determines the duration of the IAP phases. The fourth phase is not really relevant for short radial distances (from 0.05 to $0.4 \mathrm{~mm}$ ).

Default values: We shall refer to $A_{1}, A_{2}, A_{3}$ and $T_{\mathrm{spl}}$ as excitation parameters. Their values will be set to those suggested by Dimitrov and Dimitrova [2] $\left(A_{1}=21242\right.$, $A_{2}=5.0, A_{2 \mathrm{~b}}=5.0, A_{3}=14$ and $T_{\mathrm{spl}}=2.5 \mathrm{~ms}$ ), except when we vary one of them in the course of our study. These values will be called the default values for the excitation 
parameters. All our simulations were performed using a point electrode and a sample frequency of $250 \mathrm{kHz}$.

Ranges of variation: According to [10], the values for parameter $A_{2}$ are restricted to the range 2-8. Parameter $A_{3}$ has its values limited within the range 10-20 and parameter $T_{\text {spl }}$ has a range of 1.0-4.0 ms.

\subsection{Impulse response parameters for the D-D model. Default values. Setting parameters}

The D-D impulse response can be expressed as a function of four parameters, namely the radial distance, $r$, the axial distance, $z_{0}$, the fibre diameter, $d$, and the constant of anisotropy, $K_{\text {an }}[10]$.

Default values: We shall refer to $r, z_{0}, K_{\mathrm{an}}$, and $d$ as impulse response parameters and except when we vary one of them, their values will be set to $r=0.2 \mathrm{~mm}, z_{0}=20 \mathrm{~mm}$, $K_{\mathrm{an}}=5$ (as suggested by Miller-Larson [10]) and $d=0.055$ $\mathrm{mm}$. These values will be called the default values for the impulse response parameters.

Setting parameters: We consider a $90 \mathrm{~mm}$ long fibre with a right semilength of $50 \mathrm{~mm}$. We use a reference velocity of $4 \mathrm{~mm} / \mathrm{ms}$ and a time step (sampling interval) of $4 \mu \mathrm{s}(250 \mathrm{kHz})$. This means that the space step used for the simulations is $0.016 \mathrm{~mm}$, which is suitable for the radial distances of interest (0.05-0.4 mm).

Note that, although we use reference values for $d$ and $v$, we will vary their values in the course of our study to examine their effects on MSD.

SFAP dependence on the IAP

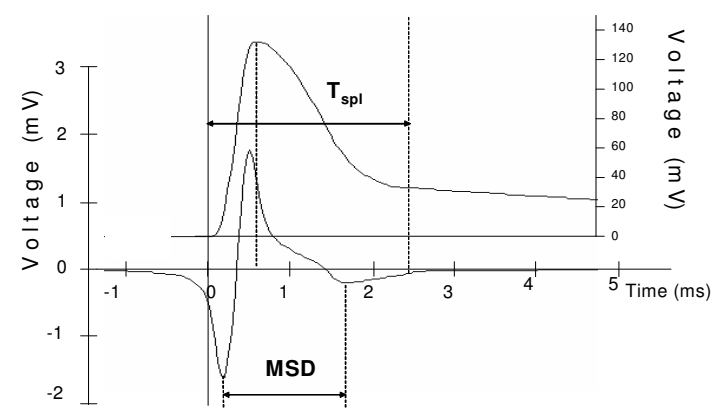

Fig. 4 Influence of the IAP phases on the generation of the SFAP phases Note that the rising phase of the IAP generates the first two phases of the SFAP whereas the 2 nd and 3 rd IAP phases (i.e. the $T_{\text {spl }}$ ) determine the terminal phase of the SFAP.

\subsection{Influence of the IAP phases on the SFAP}

In Fig. 4 we superimpose a SFAP and its corresponding IAP. In this figure, the delay introduced in the SFAP by the convolution between the IAP and the impulse response has been removed on purpose in order to show the influence of the IAP portions on the SFAP profile. Note that not all the IAP phases have the same effect on all the phases of the resulting SFAP. It can be appreciated that the first two phases of the SFAP corresponds mainly to the depolarization portion of the IAP while the third phase of the SFAP essentially corresponds to the repolarization portion of the IAP [20].

As we explain in Section 3.1, the duration of the IAP depolarization phase is fixed by parameters $A_{2}$ and $A_{3}$ (5), whereas the duration of the repolarization is basically determined by the $T_{\text {spl }}$ parameter. This means that the up- time of a SFAP is determined by $A_{2}$ and $A_{3}$ whereas the decay time is largerly determined by $T_{\mathrm{spl}}$. Since the decay time is clearly longer that the up-time (see Fig. 1), we can assume that the MSD of a SFAP is basically determined by $T_{\text {spl }}$.

Note that, in the above discussion, we have not considered the effect of the impulse response parameters on SFAP generation. In the next section, we analyze the influence of all the parameters involved in the D-D model on the MSD of a SFAP. Specifically, we study the validity of our hypothesis of MSD dependence on $T_{\mathrm{spl}}$.

\subsection{Influence of the D-D parameters on time parameters DF25, DS25, SP10, UT, DT and MSD.}

Fig. 5 shows the dependence of time parameters DF25, DS25, SP10, UT, DT and MSD on both the excitation parameters (columns one to four) and impulse response parameters (columns five to eight). In each column we only vary the value of one D-D parameter whereas the other parameters take the default values. Note that each of the rows of Fig. 5 corresponds to a different time parameter.

In the first three rows we see that the sensitivity of time parameters DF25, DS25 and DSP10 with $v$ (7th column) is of comparable magnitude to that of $r$ (8th column) and significantly more important than that of $A_{2}$ (2nd column), $A_{3}$ ( 3 rd column), $T_{\text {spl }}$ (4th column) and $K_{\text {an }}$ (5th column).

In the 4th row we see that the variation of the UT with parameters $A_{2}$ (2nd column), $A_{3}$ (3rd column), $v$ (7th column) and $r$ (8th column) has a similar weight. Note that the effect of parameter $T_{\text {spl }}$ on the UT is negligible.

In the 5th and 6th rows, however, we see that the influence of $T_{\mathrm{spl}}$ on SFAP parameters DT and MSD is significantly more important than that of $A_{2}, A_{3}, v$ and $r$. Moreover, the influence of parameters $A_{2}, A_{3} z_{0}$ and $K_{\text {an }}$ on the SFAP MSD is negligible.

\section{The special features of parameter MSD}

As we see in Fig. 1(a), parameter MSD can be computed as the sum of parameters UT and DT. As a consequence, since the variations of the UT and the DT with $A_{2}$ [Fig. 5(d2)-(e2)] and $A_{3}$ [Fig. 5(d3)-(e3)] are of similar weight but opposite sign, the sensitivity of MSD with $A_{2}$ and $A_{3}$ is very small [Fig. 5(f2)-(f3)].

Among the excitation parameters, therefore, we note that only the $T_{\text {spl }}$ parameter has a noticeable influence on parameter MSD. Specifically, as $T_{\mathrm{spl}}$ increases, MSD gets higher [Fig. 5(f4)]. Therefore, in the IAP description proposed in the D-D model, the rising phase has no effect on MSD and only the duration of the second and third phases of the IAP (i.e. $T_{\text {spl }}$ ) has a pronounced influence on it. This result is not surprising: as we explained in Section 3.3 , the IAP repolarization phase (whose duration is determined by $T_{\text {spl }}$ ) essentially generates the third phase of a SFAP, whose duration is basically the MSD.

Among the impulse response parameters, we note that only $v$ and $r$ [Fig. 5(f7)-(f8)] have a slight influence on MSD. However, their effects are very small in comparison with that of $T_{\mathrm{spl}}$. This means that although we consider a variability in the diameter of the denervated muscle fibres it is of little relevance in the variation of the MSD (this variation is studied in section 3.5). We can conclude, 
therefore, that geometry and volume conduction parameters do not significantly affect MSD.

Influence of the IAP parameters on time parameters
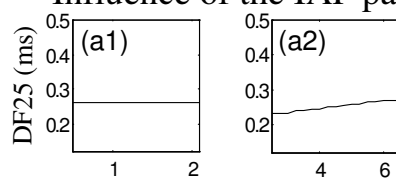

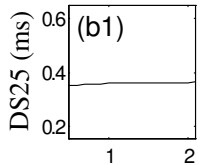
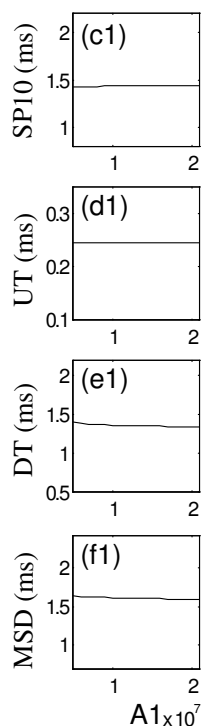
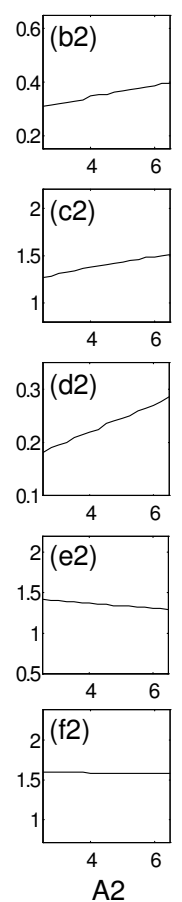
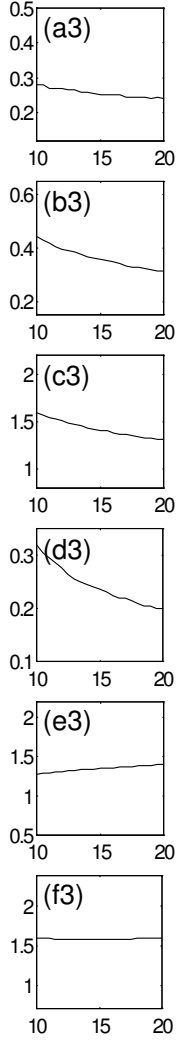

A3
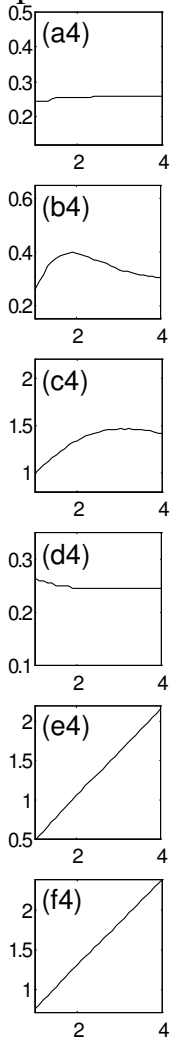

Tspl (ms)
Influence of the IR parameters on time parameters
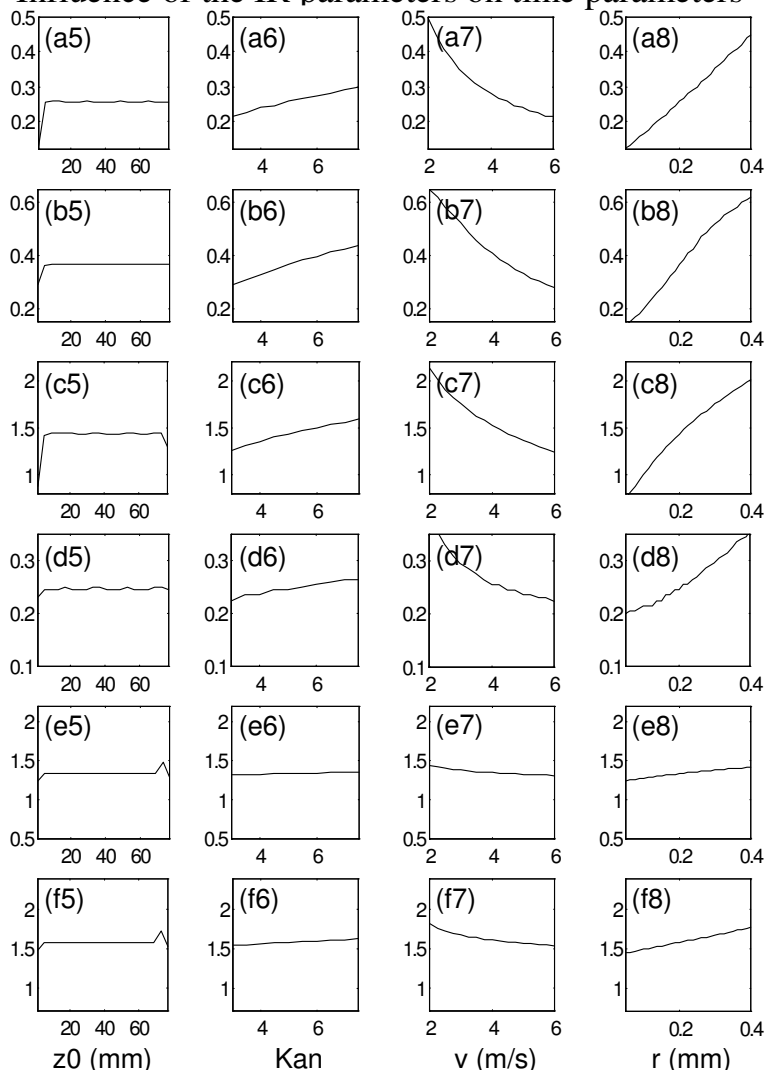

Fig. 5 Dependence of time parameters DF25, DS25, SP10, UT, DT and MSD on both the excitation parameters (columns 1-4) and impulse response parameters (columns 5-8). Each of the rows corresponds to a different time parameter. Default values are used for the non-varying parameters. The system parameters are used for the fibre length and the sample frequency. Note that, for time parameters DT and MSD, the influence of parameter $T_{\text {spl }}$ is significantly more important than that of propagation velocity and radial distance.

\section{Influence of the propagation velocity on the MSD}

The propagation velocity of the action potential in a muscle fibre varies with the temperature of the muscle. Specifically, the propagation velocity decreases when reducing the temperature [1].

The propagation velocity also depends on the muscle fibre diameter. In our study, a physiological range of 2-6 $\mathrm{m} / \mathrm{s}$ is chosen to model the variability in the propagation velocity among the fibres of ADM and EDC muscles (see the 7 th column). This study was carried out under the assumption that a linear law exists between $v$ and $d$ [see Eq. (3)]. By using this law and the given range of $v$, we obtain a range of $10-95 \mu \mathrm{m}$ for the fibre diameter.

After studying the six time parameters of Fig. 5 we conclude that only in the case of parameters DT and MSD the effect of the variation of the propagation velocity is much lower than that of the synthesis parameter $T_{\mathrm{spl}}$.

\subsection{MSD variation in the D-D model}

As we have seen, not all the parameters that appear in the D-D model affect the MSD in the same way. In fact, except for parameters $T_{\mathrm{spl}}$, propagation velocity (and, therefore, the fibre diameter) and radial distance, the rest of the D-D model parameters hardly alters the MSD [see row 6 of Fig. 5].

We now examine the MSD variation that D-D model can generate using combinations of $d$ and $r$ [Fig. 6(a)] and combinations of $T_{\text {spl }}$ and $r$ [Fig. 6(b)]. In both cases, radial distances are restricted within the range $0.05-0.40 \mathrm{~mm}$.
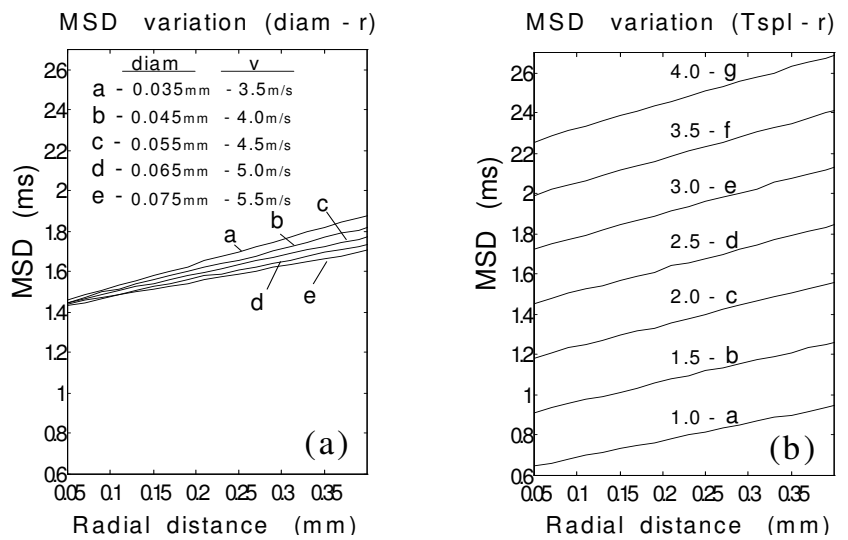

Fig. 6 (a) MSD dependence on radial distance for different values of fibre diameter. Curves $a, b, c, \mathrm{~d}$ and $e$ correspond to the fibre diameter values $35,45,55,65$ and $75 \mu \mathrm{m}$, respectively. (b) MSD dependence on radial distance for different values of $T_{\text {spl. }}$. Curves $a, b, c$ and $d$ correspond to $T_{\text {spl }}$ values of $1.0,2.0,3.0$ and 4.0 , respectively. The non-varying excitation and impulse response parameters have been set to their default values.

\section{Analysis of the combinations of $d$ and $r$}

In Fig. 6(a) we vary the fibre diameter between 0.035 and $0.075 \mathrm{~mm}$. This means that a standard deviation of $20 \mathrm{~mm}$ is assumed around the default value of $d, 0.055 \mathrm{~mm}$. Using 
(3), the latter range for the fibre diameter corresponds to a range of $3.5-5.5 \mathrm{~m} / \mathrm{s}$ for the propagation velocity.

The whole range of variation obtained for the MSD parameter using the latter distributions for $d$ and $r$ is 1.42$1.9 \mathrm{~ms}$. This range turns to be too small in comparison with the ranges observed for the ADM and EDC muscles [1.4$3.2 \mathrm{~ms}$, Fig. 2(a), and 1.0-2.3 ms, Fig. 2(b), respectively]. Therefore, although the variability in the diameter of the denervated muscle fibres has a slight effect on the SFAP MSD, it cannot completely explain the MSD variation observed in our FPs.

\section{Analysis of the combinations of $T_{\mathrm{spl}}$ and $r$}

Fig. 6(b) shows the MSD values obtained for different combinations of $T_{\mathrm{spl}}$ and $r$ parameters. In our previous study [10] we found that $T_{\text {spl }}$ should be bounded and proposed a lower and upper bounds of 1.0 and 4.0, respectively. We will use these values again.

Curve $a\left(T_{\mathrm{spl}}=1.0 \mathrm{~ms}\right)$ generates the minimum MSD values and curve $g\left(T_{\mathrm{spl}}=4.0 \mathrm{~ms}\right)$ the maximum ones. For each value of $T_{\text {spl }}$ the total MSD variation is limited to 0.4 ms. Note that this value almost reaches the whole MSD variation generated by the distribution of the fibre diameter studied before. However, a variation of $0.4 \mathrm{~ms}$ in the MSD is still too small in comparison with the experimental MSD variations obtained for the ADM and EDC muscles (1.8 and $1.3 \mathrm{~ms}$, respectively).

Therefore, if we want to reach the MSD variability of real data we should allow $T_{\text {spl }}$ to vary within a certain range. In fact, if we let $T_{\mathrm{spl}}$ to vary within the range $1.0-4.0 \mathrm{~ms}$, then we obtain an overall MSD variation of 2.0 [range 0.62.6, see Fig. 6(b)], a value close to that of the ADM and EDC muscles (see Fig. 2).

\subsection{Conclusions}

Below is a summary of the findings related to the MSD parameter in the D-D model.

a) The MSD of a SFAP basically depends on the $T_{\mathrm{spl}}$ parameter of its respective IAP.

b) Only in the case of parameters DT and MSD, the effect of the propagation velocity and other parameters is much lower than that of $T_{\text {spl }}$.

c) According to the D-D model, we should allow the $T_{\text {spl }}$ parameter to vary within a certain range in order to model the MSD variability observed in experimental recordings.

\section{Estimation of $T_{\text {spl }}$ from fibrillation potentials}

In this section, we develop a method to estimate the $T_{\text {spl }}$ value of the excitation (IAP) from the MSD value of its corresponding SFAP. This means that we are using a waveform parameter (MSD) of a SFAP to determine the value of a model parameter $\left(T_{\mathrm{spl}}\right)$. The method of estimation is grounded on the high dependence of MSD on $T_{\text {spl }}$ [Fig. 5(f4)] and the fact that the rest of parameters have a much lower influence on MSD.

\subsection{Previous considerations}

In the $T_{\text {spl }}$ estimation we do not know exactly the values of $d$ and $r$ at which the FP was recorded. This will force us to test different $d-r$ combinations and then estimate the $T_{\text {spl }}$ provided by each combination. A small error in the estimation of the $T_{\mathrm{spl}}$ arises from our uncertainty about the exact values of $d$ and $r$ and the influence of these parameters on MSD [see the curves of Fig. 6(a)]. We will measure this error later.

To provide an estimation of $T_{\mathrm{spl}}$ we will use the SFAP that best matches the FP. This means that, from the possible SFAPs synthesized using different $d-r$ combinations, we will choose the one with the profile as close as possible to that of the FP. We use the SER (signal-to-error-ratio) as the figure of merit to measure the similarity between the FP and the generated SFAP [see Eq. (7)]. We define the SER as the ratio between the energy (sum of squared signal samples) of the FP and the energy of the difference between the FP and the synthesized SFAP.

$$
S E R=\frac{\sum_{n=1}^{n=L} F P^{2}[n]}{\sum_{n=1}^{n=L}(F P[n]-S F A P[n])^{2}}
$$

From the SFAPs synthesized using the possible $d-r$ combinations we will choose the one that generates the maximum SER. We shall refer to this SFAP as SFAP matched and will take it as reference for the definite estimation of $T_{\text {spl }}$ in section 4.2 .

\section{2 $T_{\text {spl }}$ estimation}

The estimation process is presented in Fig. 7 for various combinations of $d$ and $r$. This is made to show that the value of the estimated $T_{\text {spl }}$ slightly varies depending on the values of $d$ and $r$ used to synthesized the SFAP. The $\mathrm{T}_{\mathrm{spl}}$ is estimated for all the considered cases $(r-d$ possible combinations) following the next two steps (Fig. 7):

1. First of all, we superimpose the simulated SFAP (dashed line) and the reference FP (solid line) so that their first phases are placed at the same position [see Fig. 7(a)-(e)-(i)]. We set a reference point $\mathrm{P}_{1}$ at the peak of the FP first positive (downward) phase. The simulated SFAP is aligned to the FP at $\mathrm{P}_{1}$.

2. After the first step, the third phases of the SFAP and FP are still different [Fig. 7(a)-(e)-(i)]. The MSD of the simulated SFAPs is clearly longer than that of the FP. Specifically, they differ in $\Delta t$ $\mathrm{ms}$, the time elapsed between points $\mathrm{P}_{2}$ and $\mathrm{P}_{3}$ (corresponding to the $3 \mathrm{rd}$ peak in the FP and in the SFAP, respectively). Then, we vary the $T_{\text {spl }}$ value of the synthetic SFAPs so that their MSDs approximates to that of the analysed FP [see in Fig. 7, columns 2-4 that the t-axis coordinates of $\mathrm{P}_{2}$ and $\mathrm{P}_{3}$ are equal]. This adjustment is based on the curves of Fig. 6 . The $T_{\mathrm{spl}}$ of the synthetic SFAP is taken as the $T_{\mathrm{spl}}$ estimate of the FP.

3. The final estimation of the $T_{\mathrm{spl}}$ is obtained from the SFAP that better matches the FP, that is, the $\mathrm{SFAP}_{\text {matched }}$. 
In each of the rows of Fig. 7, different SFAPs are generated using a fixed value for the fibre diameter and different values for the radial distance. Specifically, diameter values of $0.045,0.055$ and $0.065 \mathrm{~mm}$ are used in the first, second and third rows, respectively. This means that a standard deviation of $10 \mathrm{~mm}$ is used to model the variability in the diameters of the denervated fibres.
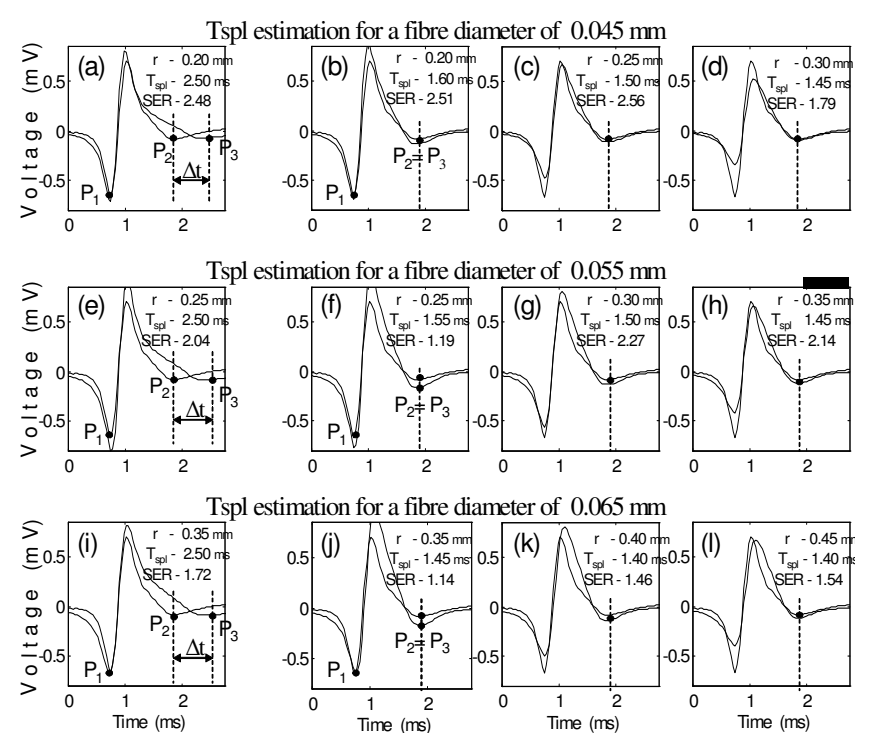

Tspl estimation for a fibre diameter of $0.065 \mathrm{~mm}$

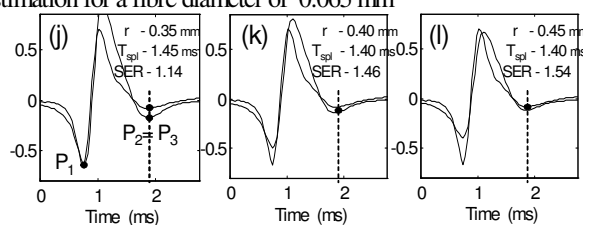

Fig. 7 Estimation of $T_{\text {spl }}$ for 9 different combinations of $d$ and $r$ (columns 2 to 4 ). The SFAPs of the 1st, 2nd and 3rd rows are generated using fibre diameter values of 45,55 and $65 \mu \mathrm{m}$, respectively. The estimation process is divided in two steps. The figures of the 1st column (a)-(e)-(i) represent the first step: sSimulated SFAPs (dashed line) and real FPs (solid line) are superimposed so that their first negative peaks have the same position. Note that the MSDs of the SFAPs and FPs are different. The figures of the 2nd, 3rd and 4th columns represent the second step: parameter $T_{\text {spl }}$ of the SFAPs has been adjusted so that their MSDs are similar to those of the FPs. Note that the estimated values for the $T_{\text {spl }}$ are different for each $d-r$ combination. The SER values are also indicated. Note that the SER value increase with the similarity between the profiles of the SFAP and the FPs.

As can be seen in Fig. 7, there is some variability in the estimation of the $T_{\mathrm{spl}}$, which depends on the $d-r$ combination used. If we look to the first row for example, we see that the estimated values for $T_{\text {spl }}$ are 1.60, 1.50 and $1.45 \mathrm{~ms}$ [Fig. 7(b)-(c)-(d), respectively]. The variability of the estimation is, therefore, $0.15 \mathrm{~ms}$. In the second row the $T_{\text {spl }}$ estimated values are $1.55,1.50$ and $1.45 \mathrm{~ms}$ [Fig. 7(f)(g)-(h), respectively] and the variability of the estimation is reduced to $0.10 \mathrm{~ms}$. Finally, in the third row the estimated values for $T_{\text {spl }}$ are 1.45, 1.40 and $1.40 \mathrm{~ms}$ [Fig. 7(j)-(k)-(l), respectively] with a variability of $0.05 \mathrm{~ms}$.

Finally, note the different SER values obtained in the figures of columns 2-4 of Fig. 7. As can be seen, those SFAPs whose morphologies are closer to that of the FP generate a higher SER value [Fig. 7(b)-(c)-(g)].

\section{Results}

By using the method described above we can estimate the $T_{\text {spl }}$ values of the fibrillation potentials we recorded from ADM and EDC muscles. Fig. 8 shows the $T_{\text {spl }}$ histograms obtained.
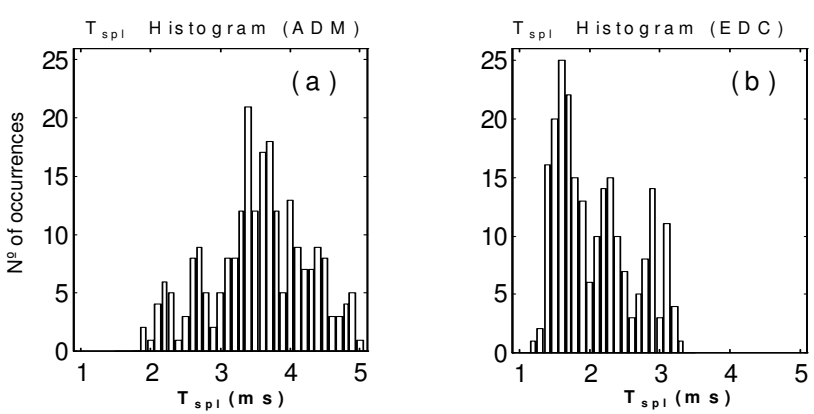

Fig. $8 T_{\text {spl }}$ histograms for the ADM (a) and EDC (b) muscles. Note the similarity in the profiles between these histograms and the MSD histograms of Fig. 2.

The most frequent $T_{\text {spl }}$ value is $3.4 \mathrm{~ms}$ for the ADM muscle and $1.6 \mathrm{~ms}$ for the EDC. Thus, as happened with the MSD histograms, the $T_{\text {spl }}$ histograms are centred around a different value for each muscle. Moreover, the dispersion around the most frequent $T_{\text {spl }}$ value is also different for each muscle. In the ADM case, most of the sample values are in the 2.8-4.2 range $(\mathrm{Std}=0.74)$ whereas for the EDC, they are between 1.2 and $2.4(\mathrm{Std}=0.59)$.

Statistical figures (mode, std and range) for the MSD and $T_{\text {spl }}$ histograms are presented in Table 1. Additionally, statistics of the SER are included.

\begin{tabular}{|c|c|c|c|c|c|c|c|c|c|}
\hline & \multicolumn{3}{|c|}{ MSD } & \multicolumn{3}{c|}{$T_{\text {spl }}$} & \multicolumn{3}{c|}{ SER } \\
\hline & Mode & Std & Range & Mode & Std & Range & Mode & Std & Range \\
\hline ADM & 2.2 & 0.37 & 1.5 & 3.4 & 0.74 & 3 & 6.5 & 2.47 & 13 \\
\hline EDC & 1.3 & 0.32 & 1.3 & 1.6 & 0.59 & 2 & 3.0 & 0.80 & 12 \\
\hline
\end{tabular}

Table 1. Estimates of MSD, $\mathrm{T}_{\mathrm{spl}}$ and SER (mode, standard deviation and range) for the whole range of variation of the ADM and EDC muscles.

Since the SER indicates the quality of the estimation for the $T_{\text {spl }}$ we see that our method provides more trustworthy $T_{\text {spl }}$ estimations for the ADM (6.5) than for the EDC (3.0). Note that the standard deviations of MSD and $T_{\mathrm{spl}}$ values are significantly larger for the ADM muscle than for the EDC. Moreover, the total range of variation for the ADM $(3 \mathrm{~ms})$ is $50 \%$ larger than that of the EDC ( $2 \mathrm{~ms})$

Note also the high resemblance in the profiles between MSD histograms [Fig. 2(a)-(b)] and $T_{\text {spl }}$ histograms [Fig. 8(a)-(b)]. In fact, for each muscle, the $T_{\text {spl }}$ histogram can be seen as a stretched and shifted replica of its MSD histogram.

In Fig. 9 we depict the $\mathrm{T}_{s p l}$ and MSD values of the fibrillation data samples in a $\mathrm{T}_{s p l}$-MSD space. (ADM data are drawn in open circles and EDC data, in filled circles).

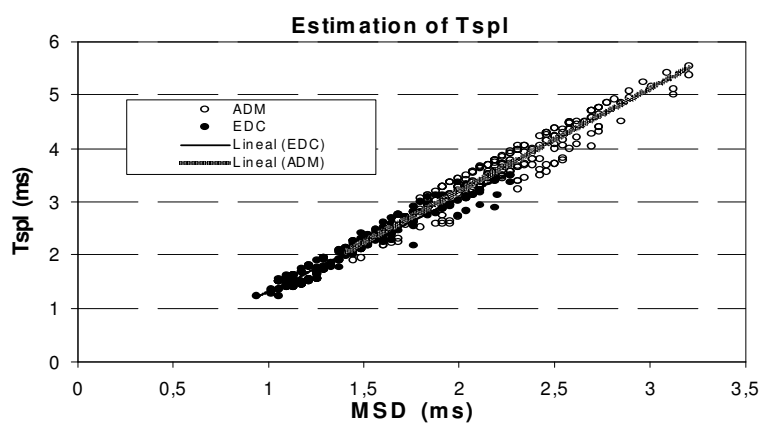


Fig. 9. Estimated $T_{\text {spl }}$ values of the FPs together with their corresponding MSD values. The relation between $T_{\text {spl }}$ and MSD parameters is almost linear. ADM samples are pictured in open circles and EDC samples are pictured in filled circles. Regression lines are plotted for each muscle separately.

Regression lines of ADM muscle and EDC muscles have very similar slopes. Furthermore, all points concentrate around their regression lines, indicating an almost linear relationship between $T_{\text {spl }}$ and MSD. This should not be surprising since the $T_{\mathrm{spl}}$ estimation is obtained from the relationships between $T_{\text {spl }}$ and MSD found in the D-D model [Fig. 6(b)]. For the case of the default values of $d$ and $r$ this relationship is almost linear [Fig. 5(f4)]. However, the samples of Fig. 9 also indicate that a statistical variability exists around the regression lines. The $T_{\text {spl }}$ estimation is carried out using the $d-r$ combination that best matches the FP and not the $d-r$ default values. The departure of this $d-r$ pair from their default values and the slight dependence of MSD on $d$ and $r$ [Fig. 6(a)] are responsible for the mentioned variability.

\section{Discussion}

Several issues arising from the previous study of the $T_{\mathrm{spl}}$ parameter deserve comment.

\section{The especial features of parameter MSD}

The time parameter MSD of a SFAP has various important features: it does not vary with any excitation parameter except with the $T_{\text {spl }}$ and presents a slight dependence on fibre diameter and radial distance. Moreover, it is the only time parameter (along with the DT) for which the effect of the propagation velocity is much lower than that of the $T_{\mathrm{spl}}$. These features make it particularly interesting for the estimation of the parameter $T_{\text {spl }}$.

\section{Variability in the $T_{s p l}$ estimation}

We have presented a method to estimate the $T_{\text {spl }}$ value of an IAP from the MSD value of its SFAP. In this method we show that the estimation for $T_{\mathrm{spl}}$ varies depending on the values of fibre diameter and radial distance used to synthesized the SFAP. To provide a final estimation of the $T_{\text {spl }}$ we take as reference the SFAP that better matches the FP.

An almost linear dependence between MSD and $T_{\mathrm{spl}}$ was found (Fig. 9). This linear relationship can be used for SFAP modelling and simulation, and as an alternative direct method for calculating the $T_{\mathrm{spl}}$ value from the MSD.

\section{Analysis of the $T_{\text {spl }}$ estimation using a concentric electrode}

The D-D model described in Section 3 assumes a point electrode, whereas we used a concentric needle electrode for recording the FPs. A concentric electrode is formed by two conductor terminals electrically isolated from each other: an inner solid wire (the "core") and an outer cycilindrical metal shaft (the "cannula"). The electrode is bevelled at the end so that the "core" is exposed at the tip showing an elliptical surface [21]. When the muscle fibre is in front of the electrode tip, the potential recorded by the "cannula" is relatively small as compared to that observed at the "core". This is particularly so in the main spike of the potential [22]-[23].

All the SFAPs presented in the paper have been simulated using a point electrode. The following two examples are provided to show that the use in the simulations of a point electrode instead of a concentric electrode does not noticeably affect the estimation of $\mathrm{T}_{\mathrm{spl}}$. In Fig. 10 we show the variability in the estimation of the $T_{\text {spl }}$ when using a different kind of electrode (a point electrode in the 1st column and a concentric electrode in the 2nd and 3rd columns). Each of the rows of Fig. 10 corresponds to a different fibrillation potential.

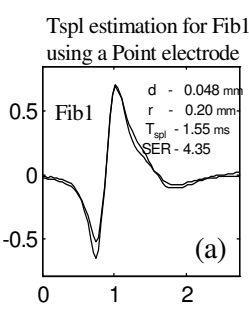

Tspl estimation for Fib2 using a Point electrode

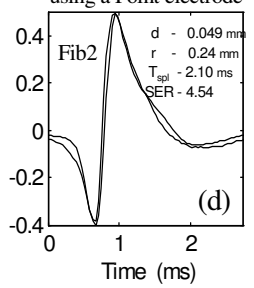

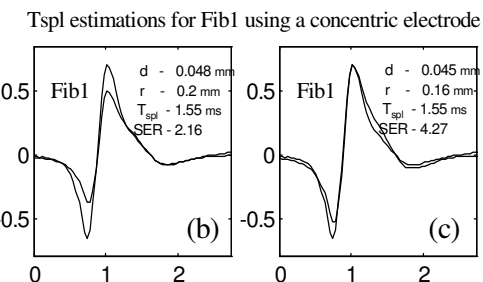

Tspl estimations for Fib2 using a concentric electrode

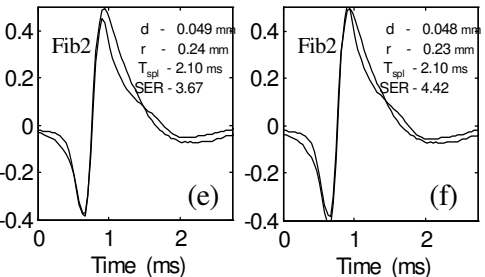

Fig. 10. Differences in the $T_{\mathrm{spl}}$ estimation using a point electrode (1st column) and a concentric electrode ( 2 nd and 3 rd columns). The study is carried out for two different FPs, Fib1 (figures in the 1st row) and Fib2 (figures in the 2nd row). For the two FPs, the estimated values for the $T_{\mathrm{sp}}$ are the same when using a point and a concentric electrode. Note that the similarity between the point electrode SFAP and the concentric electrode SFAP is larger when using larger radial distances [the simulated SFAPs (d) and (e) are more similar than SFAPs (a) and (b)]

In Fig. 10(a) we show the fibrillation potential Fib1 (solid line) and the values of $d, r, T_{\text {spl }}$ and SER obtained from the SFAP $_{\text {matched }}$ (dashed line) simulated using a point electrode. If we use a concentric electrode to simulate the SFAP [dashed line in Fig. 10(b)], then the morphology of the generated SFAP changes (note that we have kept the same values for $d$ and $r$ to appreciate this profile variation). Although the synthesized SFAP of Fig. 10(b) has considerably different its features, its MSD is very similar to that of Fig. 10(a). In fact, the $T_{\text {spl }}$ estimated value has not change. Moreover, if we vary the values of $d$ and $r$ in order to generate a SFAP that matches the FP [see Fig. 10(c)], the $T_{\text {spl }}$ estimated value remains the same.

By comparing the shape of the SFAPs of Fig. 10(a) and (b) we note that the effect of the concentric electrode on the SFAP is a smoothing and a reduction on its amplitude. This means that the use of a concentric electrode has the effect of a low pass filter. This is in connection with the fact that the potential provided by a concentric electrode is the average potential throughout the "core" surface of the tip [24].

In the second row of Fig. 10 we use a different FP, Fib2, to analyse the variability in the $T_{\mathrm{spl}}$ estimation when using a concentric electrode. Note in Fig. 10(a) that the radial distance used to synthesize the SFAP, $0.24 \mathrm{~mm}$, is slightly larger than that used for Fib1. Note also that the reduction 
of the SFAP amplitude owed to the use of a concentric electrode in Fig. 10(e) is very small. Therefore, as the radial distance increases, the dependence of the morphology of a simulated SFAP on the kind of electrode used becomes less important. Finally, the $T_{\text {spl }}$ estimated value obtained in Fig. 10(a)-(b)-(c) is the same.

The $T_{\text {spl }}$ behaviour described above has been deduced from fibrillation potentials and it is yet to be demonstrated that it accurately describes normal SFAPs. In order to confirm the accuracy of this $T_{\mathrm{spl}}$ behaviour for SFAPs, further experimental observations of single potentials taken from normal fibres are required.

\section{Conclusions}

1. According to the D-D model, the IAP repolarization phase essentially generates the terminal phase of a SFAP.

2. The MSD of a SFAP basically depends on the $T_{\mathrm{spl}}$ parameter of its respective IAP.

3. The variability in the fibre diameter and radial distance cannot completely explain the MSD variation observed in fibrillation potentials. This MSD variation can only be explained if we allow $T_{\text {spl }}$ to vary within a certain range.

4. A method for estimating the $T_{\mathrm{spl}}$ value of an IAP from the MSD of its SFAP has been proposed.

5. The relationship between MSD and $T_{\mathrm{spl}}$ has turnd out to be almost linear in our data recorded from real FPs.

6. According to our data, different muscles (ADM and EDC) have $\mathrm{T}_{\mathrm{spl}}$ values with a wide range of variation and different histogram profiles.

\section{References}

[1] S. Nandedkar, E. Stalberg. "Simulation of single muscle fibre action potentials". Med. Biol. Eng. Comput., vol. 21, pp. 158-165, 1983.

[2] G. V. Dimitrov, N. A. Dimitrova. "Precise and fast calculation of the motor unit potentials detected by a point and rectangular plate electrode", Med. Eng. Phys., vol. 20, pp. 374-381, 1998.

[3] R. Plonsey. "The active fibre in a volume conductor". IEEE Inst. Electr. Electron. Eng., vol. 21, pp. 371-381, 1974.

[4] S. Andreassen, P. Rosenfalck. "Relationship of intracellular and extracellular action potentials of skeletal muscle fibres". CRC. Crit. Rev. Bioeng., vol. 6, pp. 267-306, 1981.

[5] D. Dumitru, J. A. Delisa. "AAEM minimonograph \#10: volume conduction". Muscle Nerve., vol. 14, pp. 605-624, 1991.

[6] L. De No. "Analysis of the distribution of action currents of nerve in volume conductors". Studies from the Rockefeller Inst. Med. Res., vol. 132, pp. 384-477, 1947.

[7] J. Ekstedt. "Human single fibre action potentals". Acta Physiol Scand 61., vol. 226, pp. 1-96, 1964.

[8] M. Piotrkiewicz, A. Miller-Larsson. "A method of description of single muscle fibre activity". Biological Cybernetics., vol. 56, pp. 237-245, 1987.

[9] A. Miller-Larson. "An analysis of extracellular single muscle fibre". Biological Cybernetics., vol. 51, pp. 271-284, 1985.

[10] J. Rodríguez-Falces, A. Malanda-Trigueros, L. Gila- Useros, I. Rodríguez-Carreño, J. NavallasIrujo. "Modelling fibrillation potentials - a new analytical description for the muscle intracellular action potential". IEEE Trans Biomed Eng., vol. 53, pp. 581-592, 2006.

[11] K.C McGill, ZC Lateva. "A model of the musclefibre intracellular action potential waveform, including the slow reppolarization phase". IEEE Trans Biomed Eng., vol. 48, pp. 1480-1483, 2001.

[12] K.C McGill, ZC Lateva, S Xiao. "A model of the muscle action potential for describing the leading edge, terminal wave, and slow afterwave". IEEE Trans Biomed Eng., vol. 48, pp. 1357-1365, 2001.

K.C McGill, ZC Lateva, "Slow repolarization phase of the intracellular action potential influences the motor unit action potential". Muscle\&Nerve., vol. 23, pp. 826-828, 2000.

[14] ZC Lateva, K.C McGill, "The physiological origin of the slow afterwave in muscle action potentials". Electroencephalogr. Clin. Neurophysiolg., vol. 109, pp. 462-469, 1998.

[15] D Dumitru, JC King, WE Rogers, DF Stegeman, "Positive sharp wave and fibrillation potential modelling”. Muscle\&Nerve., vol. 22, pp. 242-251, 1999.

[16] L. R. Rabiner, R.W. Schafer. "Digital processing of speech signals". Prentice-Hall, Englewood Cliffs, New Jersey., pp. 1301-1307, 1978.

B. A. Albers, W. L. Rutten W. Wallinga. "Microscopic and macroscopic volume conduction in skeletal muscle tissue, applied to simulation of single fibre action potentials". Med. \& Biol. Eng. \& Comput., vol. 26, pp. 605-610, 1988.

[18] B. A. Albers, W. L. Rutten W. Wallinga. "Sensitivity of the amplitude of the single muscle fibre action potential to microscopic volume conduction parameters". Med. \& Biol. Eng. \& Comput., vol. 26, pp. 611-616, 1988. 
[19] G. V. Dimitrov, N. A. Dimitrova. "Extracellular potentials produced by a transition between an inactive an active regions of an excitable fibre", Electromyography clin. Neurophysiology., vol. 29, pp. 265-271, 1989.

[20] D. Dumitru. "The biphasic morphology of voluntary and spontaneous SFAPs". Muscle Nerve., vol. 17, pp. 1301-1307, 1994.

[21] J. C. King, D. Dumitru, S. Nandedkar. "Concentric and single fiber electrode spatial recording characteristics". Muscle Nerve. vol. 20, pp. 15251533, 1997.

[22] D. Dumitru, J. C. King. "Concentric needle recording characteristics related to depth of tissue penetration". Electroenceph. Clin. Neurophysiol. Vol. 109, pp. 124-134, 1998.

[23] S. D. Nandedkar, D. Dumitru, J. C. King. "Concentric needle electrode duration measurement and uptake area". Muscle Nerve. vol. 20, pp. 1225-1228, 1997.

[24] D. W. Stashuk. "Simulation of electromyographic signals". J. Electromyogr. Kinesiol., vol. 3, pp. 157-173, 1993. 